\title{
Surface structure of the CoRoT CP2 target star HD 50773 ${ }^{\star}$
}

\author{
T. Lüftinger ${ }^{1}$, H.-E. Fröhlich ${ }^{2}$, W. W. Weiss ${ }^{1}$, P. Petit ${ }^{3}$, M. Aurière ${ }^{3}$, N. Nesvacil ${ }^{1}$, M. Gruberbauer ${ }^{1}$, D. Shulyak ${ }^{1}$, \\ E. Alecian ${ }^{4}$, A. Baglin ${ }^{4}$, F. Baudin' ${ }^{7}$, C. Catala ${ }^{4}$, J.-F. Donati ${ }^{3}$, O. Kochukhov ${ }^{5}$, E. Michel ${ }^{4,6}$, N. Piskunov ${ }^{5}$, \\ T. Roudier ${ }^{3}$, and R. Samadi ${ }^{4,6}$
}

\footnotetext{
${ }^{1}$ Institut für Astronomie, Universität Wien, Türkenschanzstrasse 17, 1180 Wien, Austria e-mail: lueftinger@astro.univie.ac.at

2 Astrophysikalisches Institut Potsdam, An der Sternwarte 16, 14482 Potsdam, Germany Laboratoire d'Astrophysique de Toulouse-Tarbes, Université de Toulouse, CNRS, France

4 Observatoire de Paris, LESIA, 5 place Jules Janssen, 92195 Meudon Cedex, France Department of Physics and Astronomy, Uppsala University, 75120 Uppsala, Sweden

6 Université Pierre et Marie Curie, Université Denis Diderot, Pl. J. Janssen, 92195 Meudon, France

7 Institut d'Astrophysique Spatiale, UMR8617, Université Paris X, Bât. 121, 91405 Orsay, France
}

Received 31 March 2009 / Accepted 27 October 2009

ABSTRACT

\begin{abstract}
Aims. We compare surface maps of the chemically peculiar star HD 50773 produced with a Bayesian technique and based on high quality CoRoT photometry with those derived from rotation phase resolved spectropolarimetry. The goal is to investigate the correlation of surface brightness with surface chemical abundance distribution and the stellar magnetic surface field.

Methods. The rotational period of the star was determined from a nearly 60 days long continuous light curve obtained during the initial run of CoRoT. Using a Bayesian approach to star-spot modelling, which in this work is applied for the first time for the photometric mapping of a CP star, we derived longitudes, latitudes and radii of four different spot areas. Additional parameters like stellar inclination and the spot's intensities were also determined. The CoRoT observations triggered an extensive ground-based spectroscopic and spectropolarimetric observing campaign and enabled us to obtain 19 different high resolution spectra in Stokes parameters $I$ and $V$ with NARVAL, ESPaDOnS, and SemelPol spectropolarimeters. Doppler and Magnetic Doppler imaging techniques allowed us to derive the magnetic field geometry of the star and the surface abundance distributions of $\mathrm{Mg}, \mathrm{Si}, \mathrm{Ca}, \mathrm{Ti}, \mathrm{Cr}, \mathrm{Fe}, \mathrm{Ni}, \mathrm{Y}$, and $\mathrm{Cu}$.

Results. We find a dominant dipolar structure of the surface magnetic field. The CoRoT light curve variations and abundances of most elements mapped are correlated with the aforementioned geometry: $\mathrm{Cr}, \mathrm{Fe}$, and $\mathrm{Si}$ are enhanced around the magnetic poles and coincide with the bright regions on the surface of HD 50773 as predicted by our light curve synthesis and confirmed by photometric imaging.
\end{abstract}

Key words. stars: atmospheres - stars: chemically peculiar - stars: individual: HD 50773 - stars: magnetic field - stars: imaging

\section{Introduction}

CoRoT (Convection, Rotation and planetary Transits) is a space mission with the participation of ESA's Science Program and Research and Scientific Support Department (RSSD), Austria, Belgium, Brazil, Germany, and Spain. It focuses on high precision photometry from space, also taking advantage of observing given targets continuously during nearly half a year, which is impossible from the ground. A technical overview is presented in Boisnard \& Auvergne (2006), and the asteroseismology related mission aspects are discussed in Baglin et al. (2006) and references therein.

HD 50773 (BD -00 1488, TYC 4801-2-1, $\operatorname{mag}(B)=9.50$ ) was observed during the initial run of CoRoT only little more than a month after the launch on December 27, 2006, on board of a Soyuz Fregat II-1b. The star was chosen in the seismology field as one of ten possible targets because of its location in the classical instability strip and of its suspected chemical

* The CoRoT space mission was developed and is operated by the French space agency CNES, with participation of ESA's RSSD and Science Pograms, Austria, Belgium, Brazil, Germany, and Spain. peculiarity. The reduction of the CoRoT photometry to the N2 data format is described in Appourchaux et al. (2008), and our additional reduction steps are given in Sect. 2 of this paper.

Not much has been published about this star, which is classified in SIMBAD as an A2 star and as an A4-A9 suspected chemically peculiar star in Renson et al. (1991) with so far no measured magnetic field nor rotation period.

Magnetic Ap stars, to which HD 50773 belongs, represent about $1 \%$ to $5 \%$ of the upper main sequence stars and exhibit highly ordered, very stable and often very strong magnetic fields. They frequently show both brightness- and spectral line profile variations synchronised to stellar rotation. These variations are hitherto most successfully explained by the oblique rotator model (introduced by Stibbs 1950) and are attributed to oblique magnetic and rotation axes and to the presence of a non-uniform distribution of chemical elements on their surface.

The abundance inhomogeneities in turn are said to arise from the selective diffusion of ions under the competitive action of radiative acceleration and gravitational settling (Michaud 1970) under the influence of the (oblique) magnetic field, possibly in combination with a weak stellar wind (see, e.g., Babel 1992). 


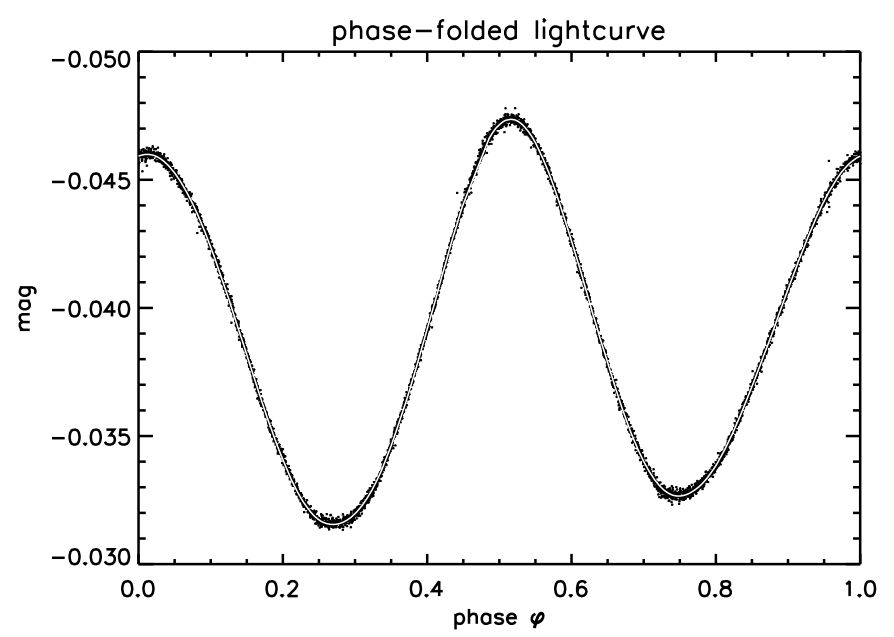

Fig. 1. Phase plot of HD 50773 using a binned light curve after the subtraction of effects due to the CoRoT orbit and the stellar signal not attributed to rotation. Overplotted (white line) is the four-spot model fit described in Sect. 4 of this paper.

The light curve of HD 50773 obtained by CoRoT has a double wave form with two clear maxima of slightly different amplitudes at phases of $\phi \approx 0.05$ and 0.52 (see Fig. 1). This photometric variability is, as mentioned, likely to be connected with the inhomogeneous surface element distribution as shown in e.g., Krtička et al. (2007) and references therein. As the star seems to be of the Cr CP2-type, we expect brighter spots rather than dark ones (see e.g. Mikulášek et al. 2007, 2008a,b). Bright photometric spots seem to be closely connected with overabundance regions of $\mathrm{Si}$ or $\mathrm{Fe}$, and the mechanism of the origin of their contrast in the optical spectrum region is well described in Krtička et al. (2009).

It is still not understood why some of the upper main sequence stars are magnetic and chemically peculiar and others are not, where the magnetic fields of CP2 stars originate from, and how these fields exactly interplay and correlate with the inhomogeneous surface distribution of chemical elements. Deriving in detail the surface structure of HD 50773 in the way presented in this paper will contribute to solving this puzzle.

Our paper is organised in Sect. 2, where we present our CoRoT space photometry, spectropolarimetric observations, and the data reduction, while the physical parameters of the target star HD 50773 and a detailed abundance analysis are presented in Sect. 3. In Sect. 4 we discuss our analysis of the surface structure of HD 50773 by applying Bayesian methods to the CoRoT light curve. Details about the magnetic field geometry and the surface abundance structures of individual elements are presented in Sects. 5 and 6. In Sect. 7 we discuss the theoretical predictions of light variability, taking into account the surface abundance inhomogeneities, and Sect. 8 is devoted to the comparison of our different approaches and the discussion of results.

\section{Observations and data reduction}

\subsection{CoRoT data}

HD 50773 was observed by CoRoT from February 3 to April 2, 2007. The reduction of the CoRoT photometry to the N2 level was performed as described in Appourchaux et al. (2008). The time span of 57.7 days covers 27.6 stellar rotational cycles, and the overall variation in brightness amounts to $0.017 \mathrm{mag}$.

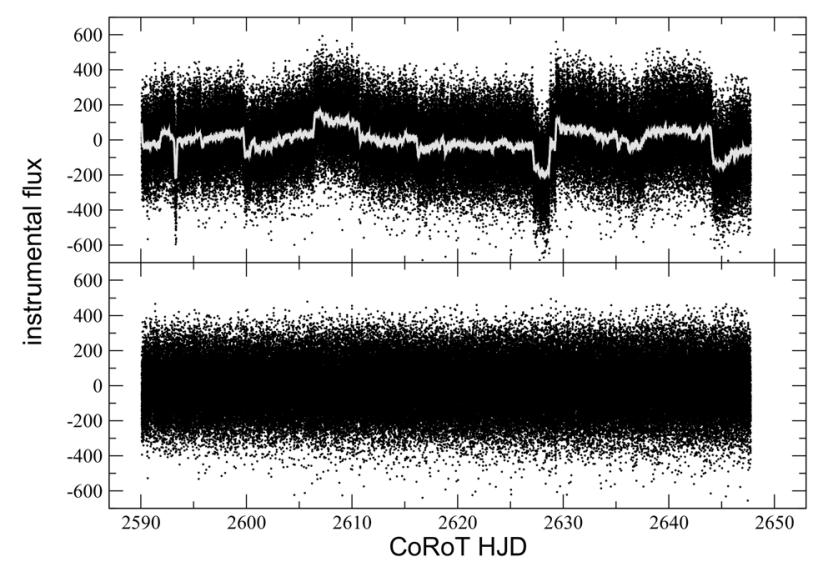

Fig. 2. Upper panel: the CoRoT light curve of HD 50773 after removal of the obvious stellar variability (black). Some small flux changes are remaining, which are shown in grey after applying a running average (see text in this section). Lower panel: after the subtraction of this running average, the residuals to the stellar variability are mostly consistent with white noise. Note that the abscissa in this plot is in CoRoT HJD (=HJD-2 451545.0) and the ordinate in CoRoT - internal flux units of the $\mathrm{N} 2$ data level after subtraction of the mean photometric signal, which is 301700 in the same units.

The wavelength range (in the CoRoT seismology field) defined by the CoRoT photometric CCD covers $2500 \AA$ to $11000 \AA$. For additonal instrumental details, we kindly refer the reader to Fridlund et al. (2006).

Any instrumental signal or stellar variation not associated with rotation and not consistent with Gaussian noise or a linear trend would affect the determination of the various parameters in our spot model. Therefore, we tested the light curve for any jumps in flux, which could either be caused by the star itself or the instrument. We first subtracted a linear trend from the time series attributed to aging effects in the detector and electronics chain (Auvergne et al. 2009) and subsequently removed the apparent stellar rotational variability from the data. Low-order polynomials were fitted to short subsets $(\sim 0.2)$ of the residual data in order to eliminate the signal changes due to rotation, which resulted in a residual time series shown in the upper panel of Fig. 2. In a next step we calculated a running average of this residual time series using a box width of 370 data points and subtracted the running average - resampled to the original time tags - from the original light curve. The result is shown in the lower panel of Fig. 2.

The upper panel of Fig. 3 shows the original CoRoT light curve with the cumulated corrections obtained so far as a thick black line. After the subtraction of these corrections we arrive at the final CoRoT light curve of HD 50773 (lower panel of Fig. 3). To reduce the large amount of 139271 data points (and the computing time) we binned the data as follows. The un-binned autocorrelation function shows a sharp drop within 0.0006 days and goes through zero at a timelag of 0.02 days. As a compromise between high time resolution - at least a fourth of CoRoT's orbital period, i.e. 0.018 days - and statistical independence, a binning length of $\approx 0.01$ days was chosen. Each timebin is represented by the median, and the assigned weight is simply the number of data used, which on average were 26.7 original data points. The binned light curve is used for our analysis described in the following sections. 
T. Lüftinger et al.: The CoRoT CP2 target star HD 50773

Table 1. Journal of the spectroscopic observations of HD 50773: dates, instrument, exposure time, peak S/N, HJD, phase, $B_{1}, \sigma$.

\begin{tabular}{cccccccc}
\hline \hline Date & Instrument & Exposure time & S/N & $\begin{array}{c}\text { HJD } \\
(2450000+)\end{array}$ & Phase & $\begin{array}{c}B_{1} \\
\mathrm{G}\end{array}$ & $\begin{array}{c}\sigma \\
\mathrm{G}\end{array}$ \\
\hline 22 Feb. 2007 & NARVAL & $4 \times 600 \mathrm{~s}$ & 136 & 4154.5095 & 0.289 & 50 & 99 \\
28 Feb. 2007 & NARVAL & $4 \times 800 \mathrm{~s}$ & 78 & 4159.5142 & 0.682 & -96 & 223 \\
28 Feb. 2007 & ESPaDOnS & $4 \times 525 \mathrm{~s}$ & 130 & 4159.7852 & 0.812 & -148 & 102 \\
04 Dec. 2007 & NARVAL & $4 \times 600 \mathrm{~s}$ & 64 & 4439.4471 & 0.557 & -179 & 188 \\
05 Dec. 2007 & NARVAL & $4 \times 600 \mathrm{~s}$ & 81 & 4439.7077 & 0.681 & -70 & 161 \\
12 Dec. 2007 & NARVAL & $4 \times 600 \mathrm{~s}$ & 156 & 4447.4335 & 0.376 & 382 & 84 \\
13 Dec. 2007 & NARVAL & $4 \times 600 \mathrm{~s}$ & 154 & 4447.6877 & 0.498 & 441 & 69 \\
13 Dec. 2007 & NARVAL & $4 \times 600 \mathrm{~s}$ & 141 & 4448.4290 & 0.852 & -386 & 94 \\
14 Dec. 2007 & NARVAL & $4 \times 600 \mathrm{~s}$ & 123 & 4448.6841 & 0.974 & -278 & 95 \\
14 Dec. 2007 & NARVAL & $4 \times 600 \mathrm{~s}$ & 163 & 4449.4622 & 0.346 & 219 & 80 \\
15 Dec. 2007 & NARVAL & $4 \times 600 \mathrm{~s}$ & 115 & 4449.7114 & 0.465 & 579 & 98 \\
15 Dec. 2007 & NARVAL & $4 \times 600 \mathrm{~s}$ & 119 & 4450.4431 & 0.815 & -45 & 119 \\
16 Dec. 2007 & NARVAL & $4 \times 600 \mathrm{~s}$ & 107 & 4450.6943 & 0.935 & -382 & 114 \\
16 Dec. 2007 & NARVAL & $4 \times 600 \mathrm{~s}$ & 110 & 4451.4378 & 0.291 & -100 & 138 \\
17 Dec. 2007 & NARVAL & $4 \times 600 \mathrm{~s}$ & 106 & 4451.6888 & 0.411 & 355 & 126 \\
18 Dec. 2007 & NARVAL & $4 \times 600 \mathrm{~s}$ & 170 & 4453.4605 & 0.258 & 9 & 77 \\
19 Dec. 2007 & NARVAL & $4 \times 600 \mathrm{~s}$ & 138 & 4453.7095 & 0.377 & 273 & 94 \\
28 Dec. 2007 & SemelPol & $4 \times 2100 \mathrm{~s}$ & 170 & 4463.1133 & 0.875 & -368 & 43 \\
01 Jan. 2008 & SemelPol & $4 \times 900 \mathrm{~s}$ & 130 & 4467.0690 & 0.766 & 13 & 64 \\
\hline
\end{tabular}

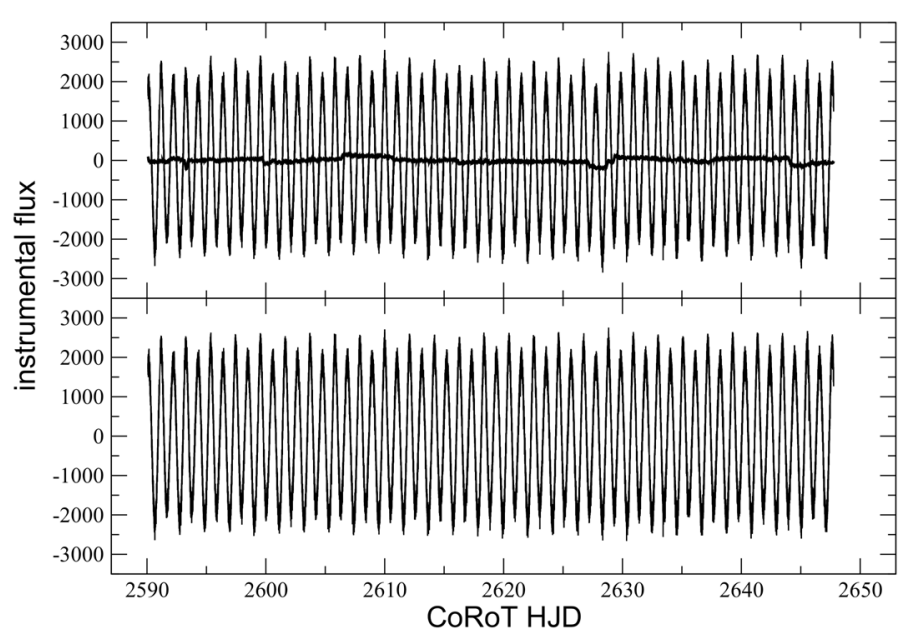

Fig. 3. Upper panel: the detrended CoRoT light curve of HD 50773 and the resampled running average containing information about residual signal obviously not related to stellar rotation (thick black line). Lower panel: the final light curve containing only signal due to stellar rotation and used for the Bayesian analysis, but zero-mean corrected for the sake of comparability. Again the abscissa in this plot is in CoRoT HJD (=HJD-2 451545.0) and the ordinate in CoRoT - internal flux units of the $\mathrm{N} 2$ data level after subtraction of the mean photometric signal, which is 301700 in the same units.

\subsection{Spectropolarimetry with NARVAL, ESPaDoNS, and SemelPol}

Spectropolarimetric observations of HD 50773 were obtained at the Canada-France-Hawaii-Telescope (CFHT) using ESPaDOnS (Donati et al. 2006) and NARVAL, which is attached to the Telescope Bernard Lyot (TBL) at Pic du Midi (February 2007, simultaneously to the COROT observations), and in December 2007 with NARVAL and SemelPol in combination with UCLES at the Anglo-Australian Telescope (AAT).

ESPaDOnS and NARVAL are twin spectropolarimeters, consisting of a Cassegrain polarimetric module and a fiberfed échelle spectrometer allowing the whole (polarimetrically analysed) spectrum from $3700 \AA$ to $10000 \AA$ to be recorded in each exposure. ESPaDOnS and NARVAL were used in polarimetric mode with a spectral resolution of $R \approx 65000$. Stokes $I$ (unpolarised) and Stokes $V$ (circularly polarised) parameters were obtained by means of four sub-exposures between which the retarders (Fresnel rhombs) were rotated in order to exchange the beams in the whole instrument and to reduce spurious polarisation signatures. The extraction of all spectra was done using Libre-ESpRIT (Donati et al. 1997), a fully automatic reduction package installed both at CFHT and TBL.

SemelPol is a visitor instrument, which is mounted at the Cassegrain focus of the AAT in combination with the UCLES spectrograph. This combination has been described in detail by e.g. Semel et al. (1993), or Donati et al. (1999, 2003). In this setup, two beams of opposite polarisation feed into the échelle spectrograph through two separate optical fibres. During the observing sequence consisting of four subexposures to obtain Stokes $I$ and $V$, the azimuth of the quarter-wave plate is switched back and forth between $+45^{\circ}$ (first and fourth exposure) and $-45^{\circ}$ (exposures 2 and 3 ), allowing for a removal of systematic erros in the measurements. Spectra cover the wavelength range between $\approx 4300 \AA$ and $6800 \AA$ with a resolving power of $R \approx 70000$. The above mentioned Libre-ESpRIT (Donati et al. 1997) package was used for data reduction.

We observed HD 50773 during 12 nights and obtained 19 Stokes $V$ (and Stokes $I$ ) series (Table 1).

For the Zeeman analysis Least-Squares Deconvolution (LSD, Donati et al. 1997) was applied to each observation. To perform the cross-correlation analysis we produced a mask calculated for physical parameters as deduced in Sect. 3 and abundances obtained in Sect. 3.2, using spectral line lists from the Vienna Atomic Line Database (VALD; Piskunov et al. 1995; Kupka et al. 1999; Ryabchikova et al. 1999). The longitudinal magnetic field $\left(B_{1}\right)$ measurements with their $1 \sigma$ error bars in $\mathrm{G}$ were computed using the first-order moment method (Rees \& Semel 1979; Donati et al. 1997).

Rotation phases of HD 50773 (Table 1) were calculated according to the ephemeris and rotation period (derived by us): $\mathrm{HJD}=2454135.09+2.09101 \times E$, where $E$ is an integer number. 


\section{Atmospheric parameters and abundance analysis}

\subsection{Atmospheric parameters}

In order to derive accurate atmospheric parameters for HD 50773, a grid of model atmospheres centered on $T_{\text {eff }}=$ $8500 \mathrm{~K}$ and $\log g=4.1$ was computed using LLMODELS (Shulyak et al. 2004). Synthetic spectra based on these models were calculated with synth3 (Kochukhov 2007) and were compared to observations. Atomic parameters used for spectrum synthesis were extracted from VALD using the default configuration file. Average surface element abundances of spectral lines corresponding to different species, which could later be used as starting values for Doppler imaging, were determined from equivalent width measurements and an adapted version of the WIDTH9 code (Kurucz 1993). For this analysis observations from different phases were co-added to reduce line profile asymmetries. The effective temperature was derived from abundance - excitation potential correlations computed for all model atmospheres in the grid using $39 \mathrm{Fe} \mathrm{I}$ and $15 \mathrm{Fe}$ II lines. This correlation is very sensitive to changes in effective temperature. For further analysis we adopted a model with $T_{\text {eff }}=8300 \mathrm{~K}$, for which the calculated $\mathrm{Fe}$ abundance was found to be independent of the excitation potential of individual transitions.

For abundance analyses of normal A type stars the surface gravity $\log g$ is usually determined via the ionisation equilibrium of the Fe lines, i.e., for a correct value of $\log g$ the iron abundance determined from a set of Fe I lines is equal to the abundance derived from Fe II lines. In the atmospheres of Ap stars, diffusion processes lead to a vertical stratification of $\mathrm{Fe}, \mathrm{Cr}$ and other elements. Spectral lines corresponding to neutral and ionised species of the same element sample different atmospheric layers. Therefore abundances determined from these two different line sets will not be equal, even for a correct choice of $\log g$. For our analysis we decided to use the value of $\log g=4.1 \pm 0.1$, based on Geneva photometry (Burki et al. 2009, in prep.).

Due to the rather high $v_{\mathrm{e}} \sin i$ of HD 50773, possible vertical abundance stratification within the stellar atmosphere had to be neglected, which leads to a minimum error of $\pm 300 \mathrm{~K}$ for $T_{\text {eff }}$.

The smallest slope in the equivalent width-abundance correlation was obtained for microturbulent velocities between 2 and $3 \mathrm{~km} \mathrm{~s}^{-1}$. This rather large value is possibly due to a combination of the effects of line broadening by the magnetic field and the co-addition of spectra from different rotation phases. Therefore, abundances obtained with $v_{\text {mic }}=2 \mathrm{~km} \mathrm{~s}^{-1}$ were used as starting values for our Doppler imaging.

\subsection{Abundance analysis}

Using the WIDTH9 code and the co-added spectrum of HD 50773, a crude abundance analysis of 17 species was performed. Due to the strong rotational variability of practically all spectral lines in this star, detailed abundances for individual rotation phases can only be derived via Doppler imaging. The values given in this section can therefore be taken only as first order approximations. In Table 2 we present abundances of 17 species derived from equivalent width measurements in co-added spectra of HD 50773, based on $T_{\text {eff }}=8300 \mathrm{~K}, \log g=4.1$, and $v_{\text {mic }}=$ $0 \mathrm{~km} \mathrm{~s}^{-1}$. For each species we give the number of lines measured and the standard deviations of the derived element abundances. A complete list of lines used for this analysis is given in Table 3.
Table 2. Abundances of 17 species derived from equivalent width measurements in co-added spectra of HD 50773.

\begin{tabular}{lccl}
\hline \hline Species & $\log N_{\text {elm }} / N_{\text {tot }}$ & \# of lines & stdev \\
\hline C I & -3.03 & 1 & - \\
Mg I & -4.49 & 2 & 0.04 \\
Si I & -4.14 & 2 & 0.22 \\
Si II & -3.93 & 2 & 0.18 \\
Ca I & -5.60 & 1 & - \\
Sc II & -8.32 & 1 & - \\
Ti II & -6.70 & 2 & 0.21 \\
V II & -6.74 & 1 & - \\
Cr I & -4.49 & 2 & 0.33 \\
Cr II & -5.10 & 7 & 0.32 \\
Mn I & -6.36 & 1 & - \\
Fe I & -4.00 & 39 & 0.30 \\
Fe II & -3.91 & 15 & 0.37 \\
Ni I & -5.48 & 1 & - \\
Y II & -9.13 & 1 & - \\
Pr III & -6.57 & 1 & - \\
Nd III & -7.82 & 1 & - \\
\hline
\end{tabular}

\section{Analysing the light curve with Bayesian methods - photometric imaging (PI)}

Fitting the light curve in terms of a few circular spots needs the estimation of many parameters. The star is described by the inclination angle $i$ and the rotational period $P$. Each spot needs at least three further parameters: longitude $\lambda$, latitude $\beta$, and spot radius $\gamma$ (in the following longitude increases in the direction of stellar rotation, and the zero point is the central meridian facing the observer at the beginning of the time series). Additional parameters are the spot intensity $\kappa$ and the coefficient $u$ in the linear limb-darkening law. We use $u=0.4415$ derived within model atmosphere calculations with the ATLAS9 code (Kurucz 1993) applying the wavelength range from $2500 \AA$ to $11000 \AA$, which is defined by the CoRoT photometric CCD.

Before analysing the data proper prior distributions have to be assigned. If information on the star's inclination $i$ is missing, $\cos i$ is evenly distributed for $0 \leq i \leq \pi / 2$. Concerning a latitude $\beta$, the value of $\sin \beta$ is assumed to be evenly distributed. All non-dimensionless parameters like radii or frequencies are represented by their logarithms. This makes certain that the posterior distribution for a radius will be consistent with that of an area and likewise the posterior for a frequency with that of a period, i.e. it does not make a difference whether one prefers radii or areas, frequencies or periods.

The likelihood function is constructed as follows. Spotted stars which are geometrically similar exhibit the same light curve, except for an offset in magnitude. This offset is considered irrelevant and removed by integration. This is even indicated, as the magnitude of the unspotted star, the zero point, is unknown. This integration can be done analytically if the measurement errors are assumed to be Gaussian-distributed over the magnitudes.

With the $N$ data points $d_{i}$ gained at times $t_{i}$, their standard deviations $\sigma_{i}$, the fit $f_{0}\left(t_{i}\right)$ and an offset $c_{0}$, the likelihood is represented by:

$$
\begin{aligned}
& \Lambda\left(\sigma_{1 \ldots N}, c_{0}, p_{1} \ldots p_{M} ; d_{i}\right)= \\
& \prod_{i=1}^{N} \frac{1}{\sqrt{2 \pi} \sigma_{i}} \exp \left(-\frac{\left(d_{i}-f_{0}\left(t_{i}, p_{j}\right)-c_{0}\right)^{2}}{2 \sigma_{i}^{2}}\right) .
\end{aligned}
$$


T. Lüftinger et al.: The CoRoT CP2 target star HD 50773

Table 3. Line list used for abundance analysis based on equivalent width measurements in the co-added spectrum of HD 50773.

\begin{tabular}{|c|c|c|c|c|c|c|c|}
\hline Species & $\lambda[\AA]$ & $E_{\text {low }}[\mathrm{eV}]$ & $\log g f$ & Species & $\lambda[\AA ̊]$ & $E_{\text {low }}[\mathrm{eV}]$ & $\log g f$ \\
\hline \multirow{2}{*}{ C I } & 6001.1179 & 8.6430 & -2.061 & Fe I & 5445.0424 & 4.3860 & -0.020 \\
\hline & & & & $\mathrm{Fe} \mathrm{I}$ & 5446.9168 & 0.9900 & -1.914 \\
\hline Mg I & 5183.6040 & 2.7170 & -0.180 & $\mathrm{Fe} I$ & 5487.7450 & 4.3200 & -0.317 \\
\hline \multirow[t]{2}{*}{$\mathrm{Mg}$ I } & 5528.4050 & 4.3460 & -0.620 & Fe I & 5554.8951 & 4.5480 & -0.440 \\
\hline & & & & $\mathrm{Fe} \mathrm{I}$ & 5565.7040 & 4.6080 & -0.213 \\
\hline Si I & 5645.6130 & 4.9300 & -1.524 & $\mathrm{Fe} \mathrm{I}$ & 5569.6181 & 3.4170 & -0.486 \\
\hline Si I & 5772.1460 & 5.0820 & -1.358 & $\mathrm{Fe} \mathrm{I}$ & 5572.8424 & 3.3960 & -0.275 \\
\hline Si I & 5948.5410 & 5.0820 & -0.780 & Fe I & 5615.6439 & 3.3320 & 0.050 \\
\hline Si II & 5055.9840 & 10.0740 & 0.593 & $\mathrm{Fe} \mathrm{I}$ & 5624.5422 & 3.4170 & -0.755 \\
\hline \multirow[t]{2}{*}{ Si II } & 5978.9300 & 10.0740 & 0.004 & Fe I & 5633.9465 & 4.9910 & -0.270 \\
\hline & & & & $\mathrm{Fe} \mathrm{I}$ & 5686.5302 & 4.5480 & -0.446 \\
\hline $\mathrm{Ca} \mathrm{I}$ & 5581.9650 & 2.5230 & -0.555 & Fe I & 5705.9922 & 4.6070 & -0.530 \\
\hline $\mathrm{Ca} \mathrm{I}$ & 5588.7490 & 2.5260 & 0.358 & Fe I & 5762.9922 & 4.2090 & -0.450 \\
\hline $\mathrm{Ca} \mathrm{I}$ & 5594.4620 & 2.5230 & 0.097 & $\mathrm{Fe} \mathrm{I}$ & 5816.3735 & 4.5480 & -0.601 \\
\hline \multirow[t]{2}{*}{$\mathrm{Ca} \mathrm{I}$} & 5857.4510 & 2.9330 & 0.240 & Fe I & 5848.1270 & 4.6080 & -1.056 \\
\hline & & & & $\mathrm{Fe} \mathrm{I}$ & 5859.5860 & 4.5490 & -0.419 \\
\hline \multirow[t]{2}{*}{ Sc II } & 5667.1490 & 1.5000 & -1.309 & Fe I & 5862.3570 & 4.5490 & -0.127 \\
\hline & & & & $\mathrm{Fe} I$ & 5914.2010 & 4.6080 & -0.131 \\
\hline Ti II & 5185.9018 & 1.8930 & -1.490 & Fe I & 5930.1799 & 4.6520 & -0.230 \\
\hline \multirow[t]{2}{*}{ Ti II } & 5418.7675 & 1.5820 & -2.000 & $\mathrm{Fe} \mathrm{I}$ & 5934.6549 & 3.9280 & -1.170 \\
\hline & & & & $\mathrm{Fe} \mathrm{I}$ & 6003.0123 & 3.8810 & -1.120 \\
\hline \multirow[t]{2}{*}{ V II } & 5819.9350 & 2.5220 & -1.703 & Fe I & 6020.1692 & 4.6070 & -0.270 \\
\hline & & & & $\mathrm{Fe} \mathrm{I}$ & 6024.0580 & 4.5480 & -0.120 \\
\hline Cr I & 5206.0370 & 0.9410 & 0.019 & $\mathrm{Fe} \mathrm{I}$ & 6027.0509 & 4.0760 & -1.089 \\
\hline Cr I & 5208.4250 & 0.9410 & 0.158 & $\mathrm{Fe} \mathrm{I}$ & 6056.0047 & 4.7330 & -0.460 \\
\hline $\mathrm{Cr}$ II & 5237.3290 & 4.0730 & -1.350 & Fe II & 5004.1950 & 10.2730 & 0.504 \\
\hline Cr II & 5310.6870 & 4.0720 & -2.280 & Fe II & 5061.7180 & 10.3080 & 0.284 \\
\hline Cr II & 5334.8690 & 4.0720 & -1.826 & Fe II & 5169.0330 & 2.8910 & -1.303 \\
\hline Cr II & 5407.6040 & 3.8270 & -2.151 & Fe II & 5234.6250 & 3.2210 & -2.230 \\
\hline Cr II & 5420.9220 & 3.7580 & -2.458 & $\mathrm{Fe}$ II & 5254.9290 & 3.2300 & -3.336 \\
\hline Cr II & 5508.6060 & 4.1560 & -2.252 & $\mathrm{Fe}$ II & 5316.6150 & 3.1530 & -1.850 \\
\hline \multirow[t]{2}{*}{$\mathrm{Cr}$ II } & 5620.6310 & 6.4870 & -1.395 & $\mathrm{Fe}$ II & 5362.8690 & 3.1990 & -2.616 \\
\hline & & & & Fe II & 5427.8260 & 6.7240 & -1.581 \\
\hline \multirow[t]{2}{*}{ Mn I } & 6021.8190 & 3.0750 & 0.034 & Fe II & 5432.9670 & 3.2670 & -3.527 \\
\hline & & & & Fe II & 5439.7070 & 6.7290 & -2.382 \\
\hline $\mathrm{Fe} \mathrm{I}$ & 5065.0180 & 4.2560 & 0.005 & Fe II & 5534.8470 & 3.2450 & -2.730 \\
\hline $\mathrm{Fe} \mathrm{I}$ & 5078.9750 & 4.3010 & -0.292 & $\mathrm{Fe}$ II & 5835.4920 & 5.9110 & -2.702 \\
\hline $\mathrm{Fe} \mathrm{I}$ & 5090.7740 & 4.2560 & -0.400 & $\mathrm{Fe}$ II & 5952.5100 & 5.9560 & -2.388 \\
\hline $\mathrm{Fe} I$ & 5133.6885 & 4.1780 & 0.140 & Fe II & 5961.7050 & 10.6780 & 0.675 \\
\hline $\mathrm{Fe} \mathrm{I}$ & 5139.4628 & 2.9400 & -0.509 & Fe II & 5991.3760 & 3.1530 & -3.540 \\
\hline $\mathrm{Fe} \mathrm{I}$ & 5162.2729 & 4.1780 & 0.020 & & & & \\
\hline $\mathrm{Fe} \mathrm{I}$ & 5202.3360 & 2.1760 & -1.838 & Ni I & 5715.0660 & 4.0880 & -0.352 \\
\hline $\mathrm{Fe} \mathrm{I}$ & 5232.9403 & 2.9400 & -0.058 & & & & \\
\hline $\mathrm{Fe} \mathrm{I}$ & 5281.7904 & 3.0380 & -0.834 & Y II & 5087.4160 & 1.0840 & -0.170 \\
\hline $\mathrm{Fe} \mathrm{I}$ & 5353.3736 & 4.1030 & -0.840 & & & & \\
\hline $\mathrm{Fe} \mathrm{I}$ & 5367.4668 & 4.4150 & 0.443 & Pr III & 6053.0044 & 0.0000 & -1.983 \\
\hline $\mathrm{Fe} \mathrm{I}$ & 5383.3692 & 4.3120 & 0.645 & & & & \\
\hline $\mathrm{Fe} \mathrm{I}$ & 5393.1676 & 3.2410 & -0.715 & Nd III & 5845.0201 & 0.6310 & -1.180 \\
\hline $\mathrm{Fe} \mathrm{I}$ & 5434.5238 & 1.0110 & -2.122 & & & & \\
\hline
\end{tabular}

Notes. Atomic parameters are taken from the VALD database. For each line wavelength, excitation potential, and log $g f$ are listed.

The unknown parameters are denoted by $p_{j}$, with $j=1 \ldots M$, with $M=24$ in the case of four spots. We set $\sigma_{i}=s_{i} \cdot \sigma$, with the relative errors $s_{i}$ being normalised according to $\sum_{i=1}^{N} 1 / s_{i}^{2}=N$.

First integrating analytically the measurement error $\sigma-$ using Jeffreys' $1 / \sigma$-prior - and then the uninteresting offset $c_{0}$, one gets a likelihood depending only on the spot modelling parameters $p_{1} \ldots p_{M}$. It is this mean likelihood, averaged appropriately over measurement error and offset, from which the posterior density distributions for all unknowns is obtained by marginalization. This is a great advantage of the Bayesian approach: it provides not only a set of most probable parameter values but moreover expectation values and error bars exclusively from the data. We can argue that the method itself determines error and offset from the data.

The Markov chain Monte Carlo (MCMC) method (cf. Press et al. 2007) has been applied to explore the likelihood mountain in a high-dimensional parameter space. The MCMC technique has already shown its capabilities when analysing photometric data from the Canadian MOST satellite (Croll 2006; Fröhlich 2007).

A set of 64 Markov chains was generated. Each chain has to perform some $10^{7}$ steps, and after a burn-in period every thou- 
Table 4. Spot parameters.

\begin{tabular}{|c|c|c|c|}
\hline \multicolumn{2}{|l|}{ Parameter } & \multicolumn{2}{|c|}{ Expectation and uncertainties } \\
\hline inclination & $i$ & $40: 3$ & $\begin{array}{l}+1.3 \\
-1.2\end{array}$ \\
\hline 1st longitude & $\lambda_{1}$ & $184: 6$ & $\begin{array}{l}-1.2 \\
+0.4\end{array}$ \\
\hline 2nd longitude & $\lambda_{2}$ & $140^{\circ} 2$ & $\begin{array}{l}-0.4 \\
+0.5 \\
-0.5\end{array}$ \\
\hline 3rd longitude & $\lambda_{3}$ & $32: 7$ & $\begin{array}{l}-0.5 \\
+0.3 \\
-0.3\end{array}$ \\
\hline 4th longitude & $\lambda_{4}$ & $312: 8$ & $\begin{array}{l}-0.3 \\
+0.3 \\
-0.3\end{array}$ \\
\hline 1st latitude & $\beta_{1}$ & 18.5 & $\begin{array}{l}-0.8 \\
+10\end{array}$ \\
\hline 2nd latitude & $\beta_{2}$ & $21: 3$ & $\begin{array}{l}-1.0 \\
+1.0 \\
-1.0\end{array}$ \\
\hline 3rd latitude & $\beta_{3}$ & $-7: 3$ & $\begin{array}{l}-1.0 \\
+0.4 \\
-0.4\end{array}$ \\
\hline 4th latitude & $\beta_{4}$ & -20.2 & $\begin{array}{l}+1.0 \\
+1.1\end{array}$ \\
\hline 1 st radius & $\gamma_{1}$ & 23.9 & $\begin{array}{l}-1.1 \\
+0.5 \\
-0.4\end{array}$ \\
\hline 2 nd radius & $\gamma_{2}$ & $15: 0$ & $\begin{array}{l}-0.4 \\
+0.5 \\
-0.5\end{array}$ \\
\hline 3rd radius & $\gamma_{3}$ & 31.3 & $\begin{array}{l}-0.5 \\
+0.4 \\
-0.5\end{array}$ \\
\hline 4 th radius & $\gamma_{4}$ & $39: 3$ & $\begin{array}{l}-0.5 \\
{ }_{-0.8}^{+0.8}\end{array}$ \\
\hline period & $P$ & 2.091007 & $\begin{array}{l}-0.80007 \\
+0.00000\end{array}$ \\
\hline spot intensity & $\kappa$ & 1.22 & $\begin{array}{l}+0.01 \\
+0.02\end{array}$ \\
\hline
\end{tabular}

Notes. We list expectation values and 1- $\sigma$ uncertainties, the period $P$ is given in days, the spot intensity is presented in units of the intensity of the unspotted surface.

sandth successful step was recorded in order to suppress the correlation between successive steps. Budding's star-spot model (1977) was used to model the light curve.

\subsection{Results from Bayesian PI}

There are two solutions which fit the light curve equally well: one with three "dark" spots and another one with four "bright" spots. In both cases the residuals amount to $0.12 \mathrm{mmag}$. The autocorrelation function of the residuals reveals that there is even more in the data than what can be represented by a simple model with circular spots. From a formal point of view the solution with "dark" spots is the more probable one, because the fit needs only three spots instead of four. But in view of the spectroscopic evidence the four-spot solution makes much more sense.

The expectation values with $1-\sigma$ confidence limits for the star and the starspot parameters are presented in Table 4. From spot longitudes, spot epochs (HJD) can be computed: $E_{i}=$ $2454135.09-2.09101 \cdot \lambda_{i} / 360^{\circ}$.

The reader should be aware that the estimated parameter values and their (surprisingly small) error bars are those of the model constrained by the data. They make sense given the model is true, i.e. that there are four circular spots, a linear limbdarkening law with prescribed coefficient holds and so forth.

In a further run the coefficient $u$ of the limb-darkening law has been determined from the data itself. This does not lead to any improvement of the fit. The fact that the deduced limbdarkening coefficient $u=0.405_{-0.013}^{+0.010}$ proves to be only a little bit smaller than the theoretical value of 0.4415 strengthens the confidence in the reliability of the four-spot model.

The high accuracy of the photometric data makes it reasonable to question the common wisdom and look for spot evolution as well as differential rotation and even for a period drift in an Ap star. Time evolution is described by a Legendre expansion of the logarithm of the spot areas up to the seventh power in time. We have done this in the case of the three dark spots only. The result is a clear null result: we neither find a spot area evolution above a 2.5- $\sigma$ level nor a hint for any departure from rigid rotation. The spot periods are constant at $\$ 3 \mathrm{ppm}$. The
Table 5. CoRoT orbit effect.

\begin{tabular}{lll}
\hline \hline Parameter & & Expectation and uncertainties \\
\hline CoRoT orbital period & $P_{\text {orbit }}$ & $103.0660 \pm 0.0012$ \\
amplitude $\left(P_{\text {orbit }}\right)$ & $A_{1}$ & $111 \pm 2$ \\
amplitude $\left(P_{\text {orbit }} / 2\right)$ & $A_{2}$ & $33 \pm 2$ \\
amplitude $\left(P_{\text {orbit }} / 3\right)$ & $A_{3}$ & $8_{-2}^{+3}$ \\
amplitude $\left(P_{\text {orbit }} / 4\right)$ & $A_{4}$ & $13 \pm 2$ \\
\hline
\end{tabular}

Notes. The estimated CoRoT orbital period is given in min, amplitudes in ppm.

lapping time for the two largest ("dark") spots would have to exceed 100 years.

It is important to clean the data from a CoRoT orbit effect: the residuals drop by a factor of five by subtracting a periodic signal containing CoRoT's orbital period as well as three overtones (Table 5).

\section{Magnetic field geometry}

All 19 Stokes $V$ LSD-line-profiles derived from ESPaDOnS, NARVAL, and SemelPol spectropolarimetric observations are grouped in a single time-series, in order to increase the rotational sampling as much as possible and build a proper data set for tomographic inversion. The time-series is then used to reconstruct the surface magnetic geometry of the star by means of Magnetic Doppler imaging (Donati \& Brown 1997).

To compute synthetic Stokes $V$ line profiles, we make a model of a spherical stellar surface divided into a grid of pixels, each pixel producing a local Stokes $I$ and $V$ profile. Assuming a given magnetic field strength and orientation for each pixel, local Stokes V profiles are calculated under the assumption, valid in the weak field limit, that Stokes $V$ is proportional to $g \cdot \lambda_{0}^{2} \cdot B_{\|} \cdot \partial I / \partial \lambda$, where $\lambda_{0}$ is the average wavelength of the LSD profile (about $534 \mathrm{~nm}$ for HD 50773), $B_{\|}$is the line-ofsight projection of the local magnetic field vector, $g$ is the effective Landé factor of the LSD profile (equal to 1.2) and $\partial I / \partial \lambda$ the wavelength derivative of the local Stokes $I$ line profile. We further assume that there are no large-scale brightness or abundance inhomogeneities over the stellar surface, so that synthetic Stokes $I$ profiles are locally the same over the whole photosphere. However we would like to mention that some features in the Stokes $V$ profile variability may arise from the influence of chemical spots and not from the magnetic topology itself, which has been demonstrated in Kochukhov et al. (2002).

For each pixel, we impose a Gaussian shape to the local synthetic Stokes $I$ line profile. Parameters of the local profile (width and depth) are chosen to optimise the adjustment of Stokes $I$ LSD profiles of HD 50773. The amplitude of the local Stokes profiles for pixels located at the visible hemisphere of the star is then weighted according to a linear limb-darkening coefficient equal to 0.49 , and their central wavelengths are shifted according to the line-of-sight velocities of individual pixels, assuming $v \sin i=46.3 \mathrm{~km} \mathrm{~s}^{-1}$ (a value minimising the $\chi^{2}$ of the magnetic model) and $i=40^{\circ}$.

Synthetic Stokes $V$ profiles are computed for the 19 observed rotation phases and are compared to the observations. The data adjustment is iterative and based on a maximum entropy algorithm (Skilling \& Bryan 1984). The version of the code used here makes a projection of the surface magnetic field onto a spherical harmonics frame (Donati et al. 2006), with the magnetic field geometry splitted between a poloidal and toroidal component 

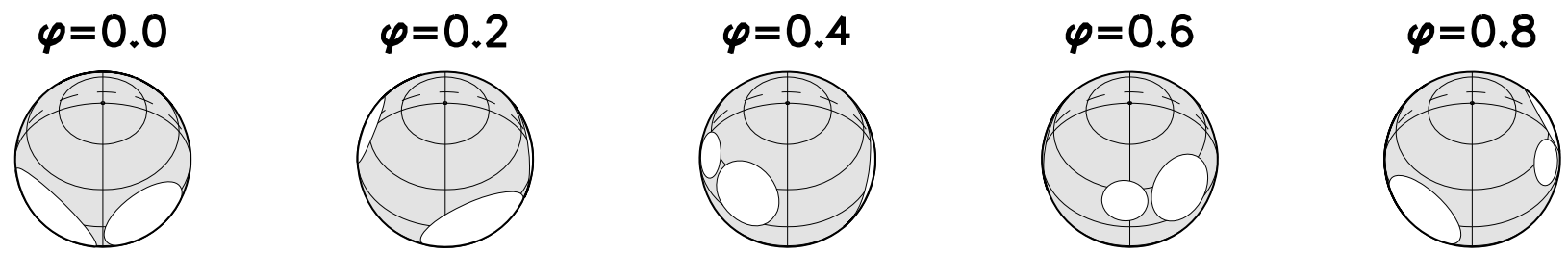

Fig. 4. Locations of the four bright photometric spots, assumed to be of circular shape, plotted at five equidistant phases.

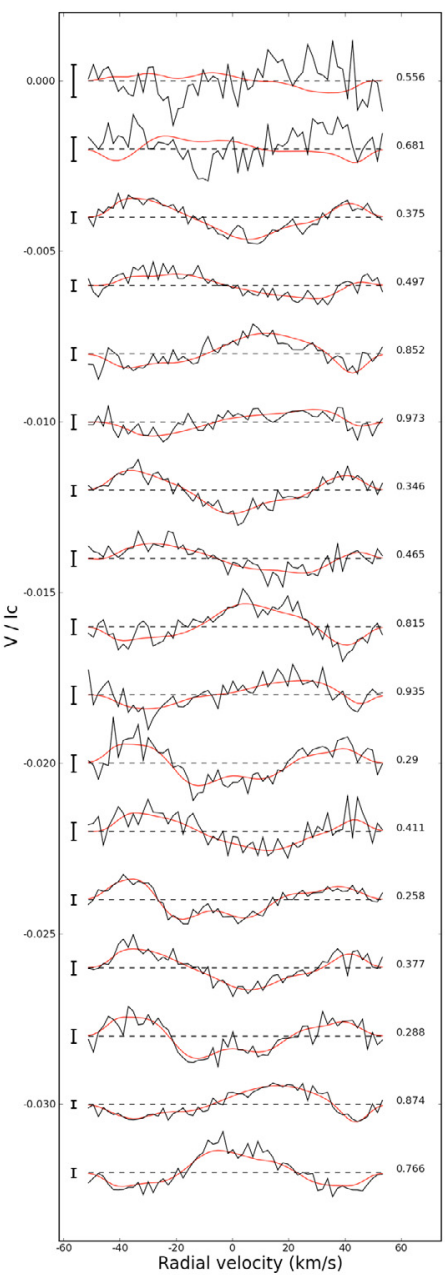

Fig. 5. Stokes V profiles of HD 50773 after the correction of the mean radial velocity of the star. Black lines represent the data and red/grey lines correspond to the synthetic profiles of our magnetic model. Successive profiles are shifted vertically for better visibility. Rotation phases of observations are indicated in the right part of the plot, and error bars are illustrated on the left of each profile.

(Chandrasekhar 1961). We limit the spherical harmonics expansion to $\ell<10$. Using this procedure, the spectropolarimetric data are adjusted at a reduced $\chi^{2}=1.2$ (Fig. 5). The final $\chi^{2}$, as well as the reconstructed magnetic topology, are left essentially unchanged as long as the adopted spherical harmonics description respects $\ell_{\max } \geq 6$. The reconstructed magnetic geometry is illustrated in Fig. 6, from which we can easily see that the surface magnetic field of HD 50773 is mostly a highly inclined dipole, with a polar strength of about $2 \mathrm{kG}$.

A closer look at the weight of the complex spherical harmonics coefficients $\alpha_{\ell, m}, \beta_{\ell, m}$, and $\gamma_{\ell, m}$ (defined by Donati et al. 2006 ) is however necessary to obtain a more quantitative information on the magnetic field distribution. Whereas the inversion
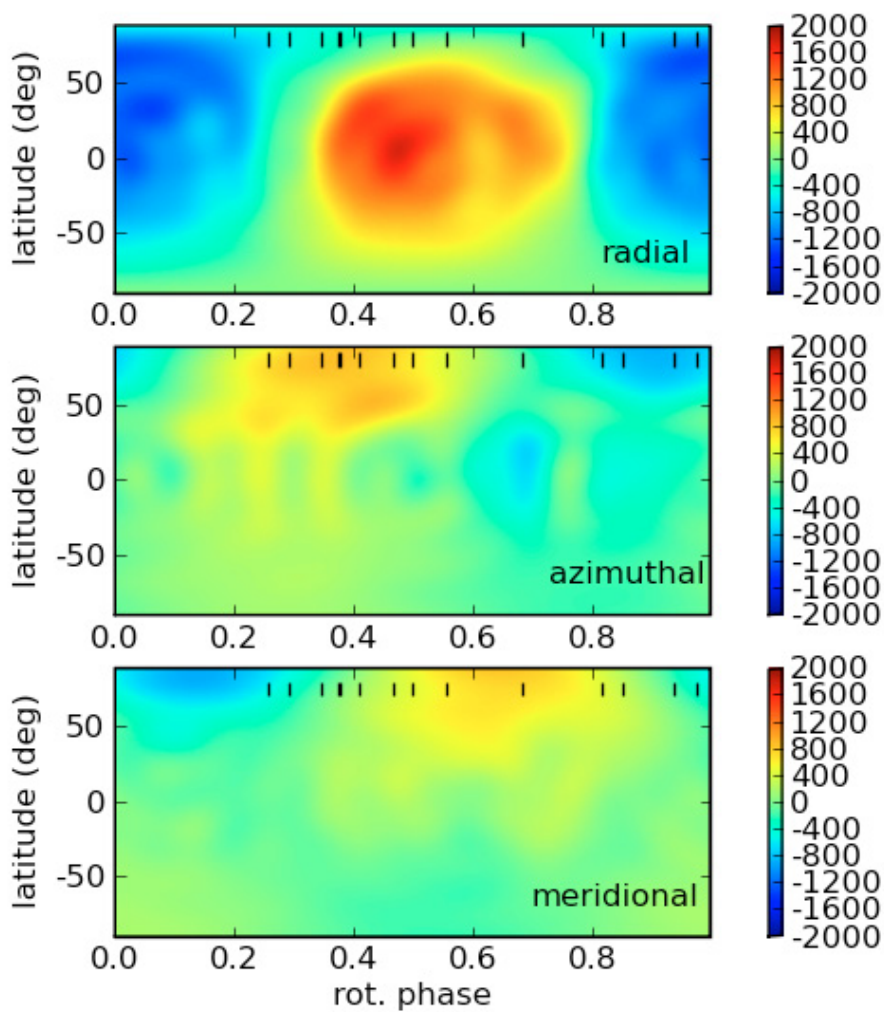

Fig. 6. Magnetic map of HD 50773, reconstructed from Magnetic Doppler imaging. Each chart illustrates the field projection onto one axis of the spherical coordinate frame with, from top to bottom, the radial, azimuthal and meridional field components. The magnetic field strength is expressed in Gauss, and the rotational phases of the observation are indicated as vertical ticks at the top of each chart.

procedure allows for the reconstruction of both poloidal and toroidal components of the field, $96 \%$ of the reconstructed magnetic energy ends up in the poloidal component. As expected for such a marginal amount of toroidal field, the outcome of the inversion code is actually almost similar if we impose the more restrictive case of a purely poloidal field. As can be readily seen on the map, most of the magnetic energy (91\%) is stored in the dipolar component. Higher-order field components are however necessary to obtain a convincing modelling of the observations (up to $\ell=6$ ). Finally, the predominance of a highly nonaxisymmetric field distribution translates into a low amount of magnetic energy in axis-symmetric spherical harmonics modes $(m=0)$, which do not gather more than $2 \%$ of the overall photospheric magnetic energy.

\section{Spectroscopic Doppler imaging}

By applying the Doppler imaging technique (DI), we are able to translate the partially very pronounced variations in the spectral line profiles of HD 50773, linked to stellar rotation, into 
Table 6. Table of elements and spectral lines used for mapping of HD 50773.

\begin{tabular}{llrrccc}
\hline \hline Species & $\lambda(\AA)$ & $\log g f$ & $E_{\text {low }}(\mathrm{eV})$ & $\begin{array}{c}\min , \max \\
\log \left(N / N_{\text {tot }}\right)\end{array}$ & Blended with & $\begin{array}{c}\odot \\
\log \left(N / N_{\text {tot }}\right)\end{array}$ \\
\hline Mg I & 5401.5210 & -0.340 & 11.6300 & & $\mathrm{Fe}, \mathrm{Y}$ & -4.51 \\
\hline Si II & 5055.9840 & 0.593 & 10.0740 & $-4.8,-2.6$ & $\mathrm{Cr}, \mathrm{Mn}$ & -4.53 \\
$\mathrm{Si}$ I & 5421.1680 & -2.250 & 5.6190 & & $\mathrm{Cr}, \mathrm{Mn}$ & \\
$\mathrm{Si}$ I & 5421.3830 & -1.480 & 5.6190 & & $\mathrm{Fe}$ & -5.73 \\
\hline Ca I & 6102.7230 & -0.862 & 1.8790 & $-6.5,-2.7$ & $\mathrm{Fe}$ & \\
$\mathrm{Ca}$ II & 6456.8750 & -0.539 & 8.4380 & & $\mathrm{Cr}, \mathrm{Fe}$ & -7.14 \\
\hline Ti II & 5154.0680 & -1.750 & 1.5660 & $-8.3,-6.1$ & & -6.40 \\
\hline Cr II & 5237.3290 & -1.350 & 4.0730 & $-7.1,-3.6$ & & \\
& 5280.0540 & -2.316 & 4.0740 & & $\mathrm{Fe}, \mathrm{Y}, \mathrm{Ni}$ & \\
& 5510.7020 & -2.614 & 3.8270 & & & -4.59 \\
\hline Fe I & 5383.3692 & 0.645 & 4.3120 & $5.4,-3.6$ & & \\
& 5400.5022 & -0.160 & 4.3710 & & & \\
& 5400.6560 & -2.482 & 3.6350 & & & \\
\hline $\mathrm{Ni}$ I & 5401.2689 & -1.920 & 4.3200 & & & \\
\hline Y II & 5510.0030 & -0.900 & 3.8470 & $-6.1,-5.6$ & $\mathrm{Cr}, \mathrm{Fe}, \mathrm{Y}$ & -5.81 \\
\hline $\mathrm{Cu}$ II & 569.8950 & -1.010 & 0.9920 & $-9.9,-7.5$ & $\mathrm{Cr}, \mathrm{Fe}, \mathrm{Ni}$ & -9.83 \\
\hline
\end{tabular}

Notes. Element, spectral lines used, the $\log g f$ values of these lines, abundance interval in dex, for comparison the solar values (Asplund et al. 2005) are presented. Atomic parameters used in our study were extracted from VALD.

surface maps of the abundance distribution. The longitude of a spot is directly deduced from the wavelength position of the distortion within the profile, whereas its latitude can only be derived from time-series observations. In the case of HD 50773, 19 such spectra (listed in Table 1) could be used for the inversions with INVERS12, the DI code we used (Kochukhov et al. 2004b). In this code, where specific intensities are calculated for each visible surface element for each iteration, it is possible to simultaneously calculate abundance maps of several chemical elements even from blended spectral lines. Mapping HD 50773, we could derive surface abundance distributions for $\mathrm{Mg}, \mathrm{Si}, \mathrm{Ca}, \mathrm{Ti}$, $\mathrm{Cr}, \mathrm{Fe}, \mathrm{Ni}, \mathrm{Y}$ and $\mathrm{Cu}$. We have to mention that Ti and Ni were derived from spectral line blends where these elements contribute only moderately to the overall absorption. Input parameters were determined as described in Sect. 3, $v_{\mathrm{e}} \sin i$ and inclination $i$ used as in Sect. 5.

\subsection{Surface abundance structures of individual elements}

\subsubsection{Magnesium and calcium}

The surface abundance distribution of magnesium was modeled using a blend of Mg I with Fe I and Y II around $5400 \AA$. We find a variation between $\log N_{\mathrm{Mg}} / N_{\text {tot }}=-5.2$ and -3.3 dex and the element, contrary to the other mapped species, does not exhibit a clear correlation with the magnetic field geometry, and is enhanced in a spotted, belt-like structure near the rotational equator (Fig. 8). The abundance distribution of calcium, variable between $\log N_{\mathrm{Ca}} / N_{\text {tot }}=-6.6$ and $-2.7 \mathrm{dex}$ and determined from $\mathrm{Ca}$ I and II blended with Fe I around $6102 \AA$ and $6456 \AA$ also shows a belt-like structure at the rotational equator with a pronounced region of overabundance at the positive magnetic pole.

\subsubsection{Iron-peak elements}

A blend of titanium II with Fe I and Cr II at $5169 \AA$ was used to recover the surface abundance structure of this element. Ti II shows a tendency to accumulate at the magnetic poles and to avoid the magnetic equatorial regions, varying between $\log N_{\mathrm{Ti}} / N_{\text {tot }}=-8.3$ and $-6.1 \mathrm{dex}$.

Chromium was mapped using several single $\mathrm{Cr}$ lines at $5237 \AA$ and $5280 \AA$ plus a blend of Cr II with Fe I Y II and Ni I around $5510 \AA$ and was found to be variable between $\log N_{\mathrm{Cr}} / N_{\text {tot }}=-6.8$ and -3.0 dex. The abundance distribution of the element shows as that of Fe a clear correlation to the magnetic field geometry, being enhanced at the poles and depleted at the magnetic equator, whereby the two enhancement regions seem to be subdivided into two spots.

The surface abundance of iron was determined using single Fe I lines at $5383 \AA$ and $5400 \AA$ and again the blend of Fe I with Cr II, Y II, and Ni I at $5510 \AA$. As mentioned above, Fe is, as $\mathrm{Cr}$, closely correlated with the magnetic field, avoiding the magnetic eqatorial region. The elemental abundance varies between $\log N_{\mathrm{Fe}} / N_{\text {tot }}=-5.3$ and -1.9 dex.

Nickel, derived from the blend of this element with Cr II, Fe I, and Y II at $5510 \AA$ and variable between $\log N_{\mathrm{Ni}} / N_{\text {tot }}=-6.1$ and -5.6 dex shows an opposite correlation to the magnetic field geometry from what we saw from $\mathrm{Cr}$ and $\mathrm{Fe}$ : it is enhanced at the magnetic equatorial regions and depleted where the positive and the negative magnetic pole cross the line of sight.

\subsubsection{Yttrium and copper}

A similar distribution as that for $\mathrm{Ni}$ can be observed for yttrium. Varying from $\log N_{\mathrm{Y}} / N_{\text {tot }}=-9.9$ to $-7.7 \mathrm{dex}$ it also shows a pronounced region of overabundance at the magnetic equator and depletion on the poles. The element was mapped from a blend with Cr II, Fe I, and Ni I at $5510 \AA$ and $5662 \AA$. Copper around $5153 \AA$, blended with Cr II, Ti II, and Fe I was used to derive the surface abundance of this element, and we see it to vary between $\log N_{\mathrm{Cu}} / N_{\text {tot }}=-8.0$ and -6.9 dex. At first glance, it seems to be closely related to the distributions of $\mathrm{Ni}$ and $\mathrm{Y}$, being enhanced at the magnetic equator, but it exhibits in addition two less pronounced spots close to the magnetic poles. 

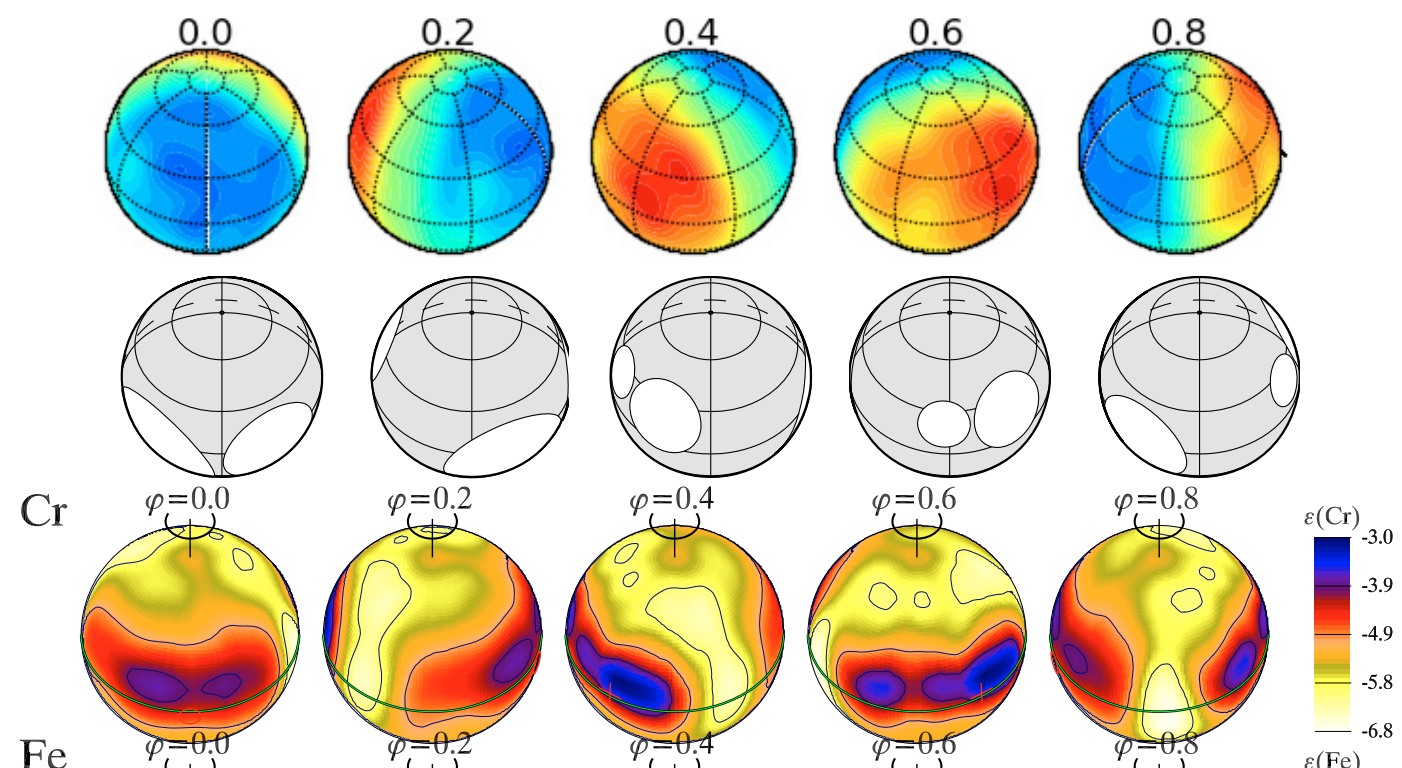

$\mathrm{Fe}$
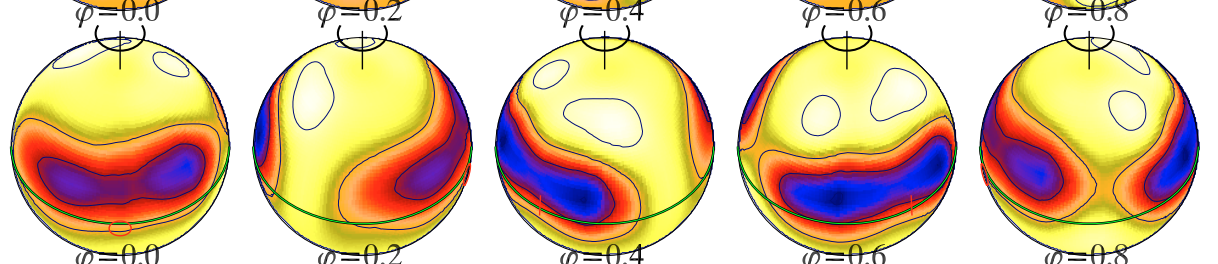

$\varepsilon(\mathrm{Fe})$

$\mathrm{Si}$
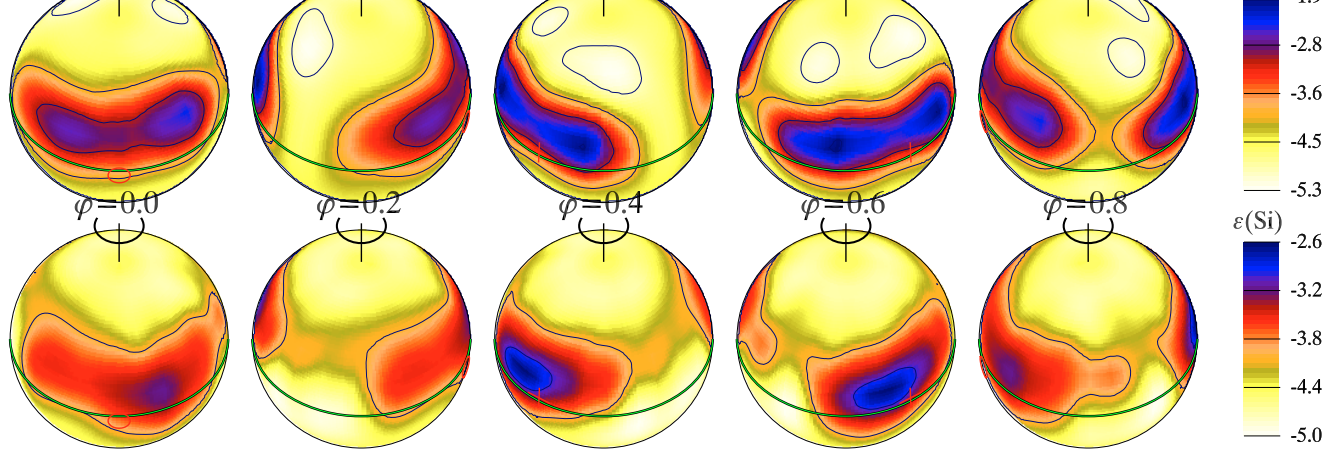

Fig. 7. Top panel: radial field component of the magnetic map of HD 50773 (as described in Sect. 5). Second panel: locations of the four bright photometric spots, assumed to be of circular shape. Next three panels: abundance distribution of $\mathrm{Cr}$, Fe, and Si at the surface of HD 50773 obtained from the lines listed in Table 6. We show the star at an inclination $i=40^{\circ}$. Darker areas in the plots correspond to higher elemental abundances, the corresponding scale is given to the right of each panel, and the contours of equal abundance are plotted with steps of 1.0 dex. The circle and the cross indicate the position of the negative and the positive magnetic pole, respectively. All projections are plotted at five equidistant rotation phases.

\section{Effect of abundance inhomogeneities on spectral energy distribution}

As already mentioned in Sect. 1 the light curve of HD 50773 obtained by CoRoT has a periodic form with two clear maxima of slightly different atmplitudes at phases $\phi \approx 0.05$ and 0.52 (see Fig. 1). This photometric variability is likely to be connected with inhomogeneous surface element distributions, similar to those recently reported and successfully modeled by Krtička et al. (2007) for the hot CP2 star HD 37776. The physical nature of this effect is directly connected with the radiative flux redistribution due to enhanced or deficient opacity in the abundance spots relative to the rest of the stellar surface. Hence, as a star rotates, the observer sees different stellar regions that are emitting a different amount of radiative flux producing characteristic variability of indices in phase-resolved photometry.

Of course, abundance spots are not the only effect which may produce visible rotational modulation in photometry. For instance, strong magnetic fields may also influence the total radiative flux gradients along the stellar surface since the magnetic opacity and flux distribution strongly depend upon the modulus of the magnetic field (Kochukhov et al. 2005; Khan \& Shulyak 2006). However, the mean surface magnetic field of HD 50773 is too weak to noticeably affect the integrated opacity coefficient, and thus the chemical spots are the most probable source of the observed variability. Details of our modelling of the light variability taking into account surface abundance variations of HD 50773 are presented in this section.

Having produced abundance maps using the Doppler imaging technique it is, in principle, possible to directly model the light curve variability in the same manner as it was done in Krtička et al. (2007). However, in case of HD 50773, there are several elements that play a noticeable role in the flux redistribution, which would require the calculation of hundreds of model atmospheres for an accurate surface integration. These extensive computations are out of the scope of the present paper. Nevertheless, to explore the role of the mapped elements on the total energy balance in the atmosphere of HD 50773 (and thus on the ability of these elements to affect the radiative balance) we computed a set of model atmospheres with different assumptions about abundance patterns. To carry out such calculations we employed the LLMODELS stellar model atmosphere code (Shulyak et al. 2004), which incorporates treatment of individual abundance patterns and computes the line opacity in a fine frequency grid for better integration of the radiation field quantities. 


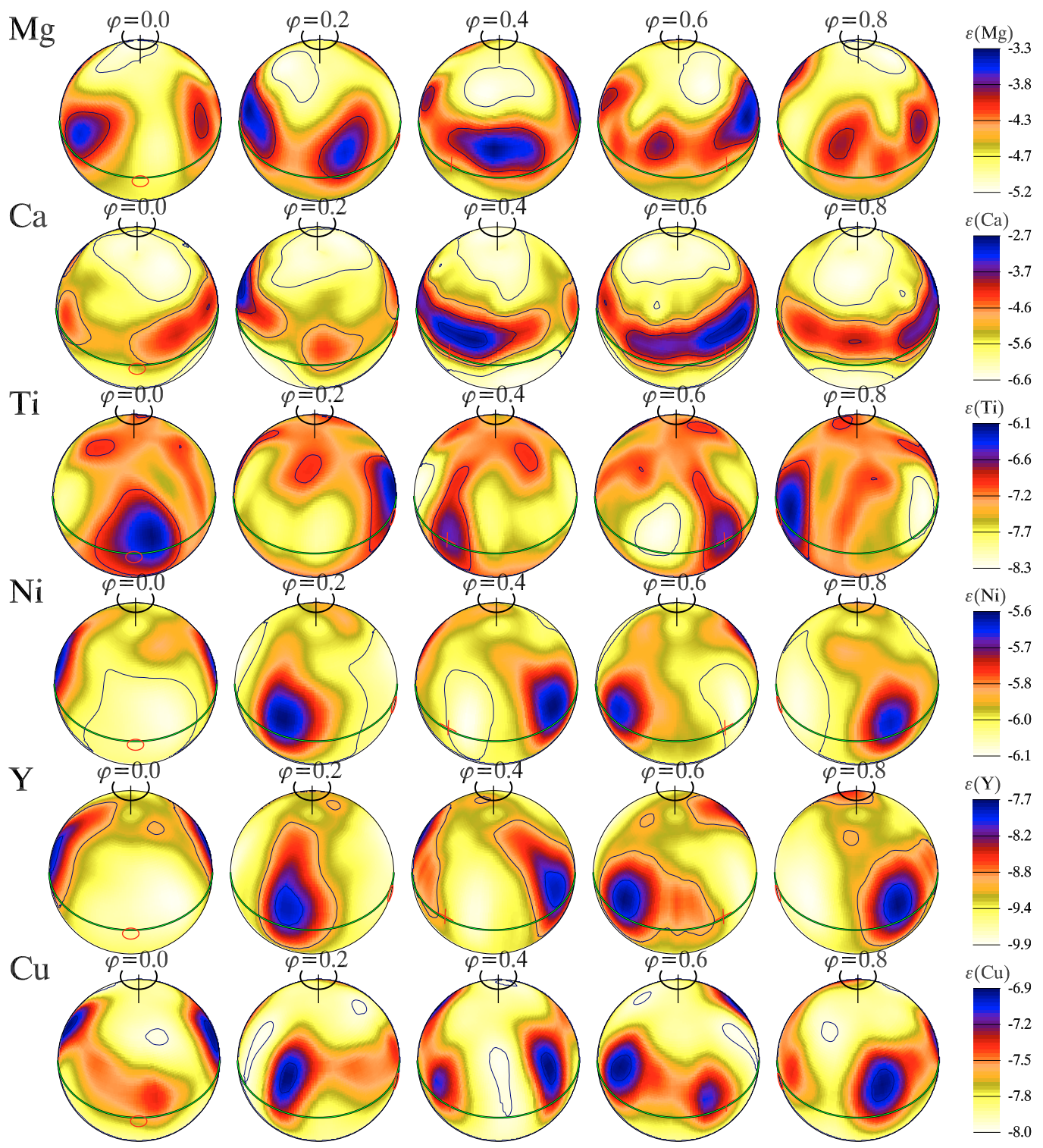

Fig. 8. Same as the three bottom panels in Fig. 7 but for $\mathrm{Mg}, \mathrm{Ca}, \mathrm{Ti}, \mathrm{Ni}, \mathrm{Y}$, and $\mathrm{Cu}$.

First we computed the model with the surface averaged abundances of all nine elements used in our DI analysis. Then another seven models were computed, individually increasing every element by +2 dex relative to its mean value. Once these computations were done, we integrated the output radiative fluxes from all the models and then compared them to the flux produced by the mean abundance reference model. The flux integration was performed in the wavelength range defined by the CoRoT photometric CCD, i.e. from $2500 \AA$ to $11000 \AA$, with the resolution of $0.1 \AA$, which is the default flux resolution in LLMODELS. The effective temperature of all the models was kept to be the same.

We find that among the nine elements considered in this investigation only four have a clear influence on the model energy distribution by more than $1 \%$. These elements are $\mathrm{Cr}(1.16 \%), \mathrm{Fe}(5.63 \%), \mathrm{Mg}(3.02 \%)$, and $\mathrm{Si}(2.21 \%)$. The influence of the other five elements is much less pronounced like, e.g. Ca $(0.22 \%)$. This agrees well with the recent study of Khan \& Shulyak (2007) who investigated the effect of individual chemistry on properties of $\mathrm{CP}$ stars and showed the relative importance of $\mathrm{Fe}, \mathrm{Cr}$, and $\mathrm{Si}$ opacity for all the models considered.
As an example, Fig. 9 demonstrates the synthetic energy distribution computed with enhanced abundances of $\mathrm{Cr}, \mathrm{Fe}, \mathrm{Mg}$, and $\mathrm{Si}$. In particular, one can note the strong impact of $\mathrm{Fe}$ and $\mathrm{Si}$ on the shape of the energy distribution. However, the relatively small changes in integrated flux values listed above are due to the fact that the CoRoT photometry covers a wide wavelength region where the flux changes due to enhanced abundance models which act in opposite directions in the UV and the visual regime. We do not show fluxes produced by the other mapped elements since they lie extremely close to the flux of the mean abundance model (thick full line).

\section{Conclusions}

The high-quality CoRoT data enabled us by applying the Bayesian data analysis to derive stellar surface structures from space quality photometry. Analysing an extensive set of ground based spectropolarimetric data via Doppler imaging and Magnetic Doppler imaging made it possible to directly correlate the results from photometry to the chemical and magnetic stellar 


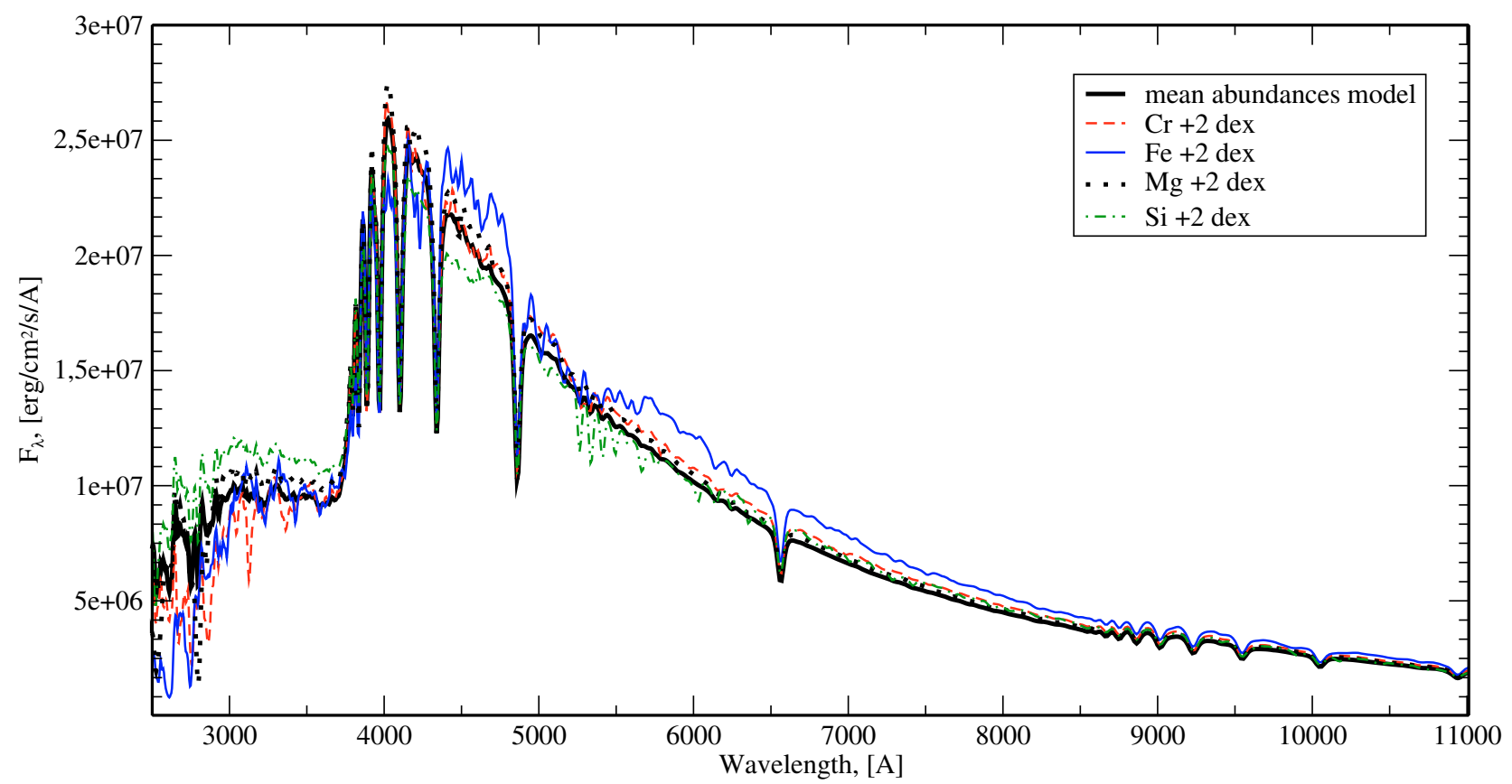

Fig. 9. The synthetic energy distributions of HD 50773 calculated with mean surface abundances and abundances of $\mathrm{Cr}, \mathrm{Fe}, \mathrm{Mg}$, and $\mathrm{Si}$ enhanced by 2 dex. For all models $T_{\text {eff }}=8300 \mathrm{~K}$ and $\log g=4.1$ were assumed. To provide a better view all fluxes were convolved with the resolution of $R=200$.

surface structure. The resulting abundances were used to model the light variability of HD 50773.

The two areas covered by bright spots found with Bayesian data analysis of the light curve coincide very well with the magnetic polar regions on the surface of HD 50773.

The elemental abundance spots of the species contributing dominantly to the changes in the integrated flux $(\mathrm{Cr}, \mathrm{Fe}$, and $\mathrm{Si})$ are also clearly correlated with the magnetic field geometry and hence with photometric spots. Regions of overabundance are found close to the magnetic poles and those of depletion linked to the magnetic equator. As described in Sect. 7, these elements produce bright spots in the photosphere.

Our results confirm the high potential of combining high quality photometric data obtained in space with ground based spectroscopy. Further studies with a similar approach are currently ongoing and will significantly increase the sample of stars analysed in this way, which is important for modelling elemental diffusion in stars in the presence of a magnetic field.

Acknowledgements. We would like to thank the referee, Z. Mikulášek, for very consctructive comments during the refereeing process. We also thank the CoRoT, CFHT and TBL teams for their observing support and the excellent data, and N. Letourneur and J.-P. Michel, who obtained the NARVAL spectra for us. This work was supported by the Austrian Science Fund FWF-P17580N2, the Lise Meitner grant No. M998-N16 to DS and by the financial contributions of the Austrian Agency for International Cooperation in Education and Research (WTZ CZ-11/2008). O.K. is a Royal Swedish Academy of Sciences Research Fellow supported by a grant from the Knut and Alice Wallenberg Foundation.

\section{References}

Asplund, M., Grevesse, N., \& Sauval, A. J. 2005, ASP Conf. Ser., 336, 25 Appourchaux, T., Michel, E., Auvergne, M., et al. 2008, A\&A, 488, 705 Auvergne, M., Bodin, P., Boisnard, L., et al. 2009, A\&A, 506, 411 Baglin, A., Michel, E., Auvergne, M., et al. 2006, in ESA SP, 1306, 3950 Babel, J. 1992, A\&A, 258, 449

Boisnard, L., \& Auvergne, M. 2006, Proceedings of The CoRoT Mission PreLaunch Status - Stellar Seismology and Planet Finding, ed. M. Fridlund, A. Baglin, J. Lochard, \& L. Conroy, ESA SP-1306, 19 Budding, E. 1977, Ap\&SS, 48, 207
Croll, B. 2006, PASP, 118, 1351

Donati, J.-F., Semel, M., Carter, B. D., et al. 1997, MNRAS, 291, 658

Donati, J.-F., Collier Cameron, A., Hussain, G. A. J., \& Semel, M. 1999, MNRAS, 302, 437

Donati, J.-F., Cameron, A. C., Semel, M., et al. 2003, MNRAS, 345, 1145

Donati, J.-F., Howarth, I. D., \& Jardine, M. M. 2006, MNRAS, 370, 629

Fridlund, M., Baglin, A., Lochard, J., \& Conroy, L. 2006, The CoRoT Mission, ESA SP-1306

Fröhlich, H.-E. 2007, Astron. Nachr., 328, 1037

Khan, S. A., \& Shulyak, D. V. 2006, A\&A, 448, 1153

Khan, S. A., \& Shulyak, D. V. 2007, A\&A, 469, 1083

Kochukhov, O. 2007, Physics of Magnetic Stars, ed. I. I. Romanyuk, \& D. O. Kudryavtsev, 109

Kochukhov, O., \& Piskunov, N. 2002, A\&A, 388, 868

Kochukhov, O., Drake, N. A., Piskunov, N., \& de la Reza, R. 2004, A\&A, 424, 935

Kochukhov, O., Khan, S., \& Shulyak, D. 2005, A\&A, 433, 671

Krtička, J., Mikulášek, Z., Zverko, J., \& Žižåovský, J. 2007, A\&A, 470, 1089

Krtička, J., Mikulášek, Z., Henry, G. W., et al. 2009, A\&A, 499, 56

Kupka, F., Piskunov, N., Ryabchikova, T. A., Stempels, H. C., \& Weiss, W. W. 1999, A\&AS, 138, 119

Kurucz, R. L. 1993, Kurucz CD-ROM, Cambridge, MA, Smithsonian Astrophysical Observatory, December 4, sssp.book

Mikulášek, Z., Krtička, J., Zverko, J., et al. 2007, in Physics of Magnetic Stars, ed. I. I. Romanyuk, \& D. O. Kudryavtsev, Special Astrophys. Obs., Nizhnij Arkhyz, 300

Mikulášek, Z., Gráf, T., Krtička, J., Zverko, J., \& Žižňovský, J. 2008a, Contr. Astron. Obs. Sk. Pleso, 38, 363

Mikulášek, Z., Krtička, J., Henry, G. W., Zverko, J., \& Žižåovský, J. 2008b, A\&A, 485, 585

Michaud, G. 1970, ApJ, 160, 640

Piskunov, N. E., Kupka, F., Ryabchikova, T. A., Weiss, W. W., \& Jeffery, C. S. 1995, A\&AS, 112, 525

Press, W. H., Teukolsky, S. A., Vetterling, W. T., \& Flannery, B. P. 2007, Numerical Recipes, 3rd edn (Cambridge: Cambridge Univ. Press)

Renson, P., Gerbaldi, M., \& Catalano, F. A. 1991, A\&AS, 89, 429

Ryabchikova, T. A., Piskunov, N. E., Stempels, H. C., Kupka, F., \& Weiss, W. W. 1999, Phys. Scr, T83, 162

Rees, D. E., \& Semel, M. 1979, A\&A, 74, 1

Semel, M., Donati, J.-F., \& Rees, D. E. 1993, A\&A, 278, 231

Shulyak, D., Tsymbal, V., Ryabchikova, T., Stütz, Ch., \& Weiss, W. W. 2004, A\&A, 428, 993

Stibbs, D. W. N. 1950, MNRAS, 110, 410 